\title{
Effect of Sequence-Dependent Rigidity on Plectoneme Localization in dsDNA
}

\author{
Shlomi Medalion and Yitzhak Rabin \\ Department of Physics and Institute of Nanotechnology and Advanced Materials, Bar-Ilan University, Ramat-Gan 52900, Israel
}

(Dated: July 9, 2018)

\begin{abstract}
We use Monte-Carlo simulations to study the effect of variable rigidity on plectoneme formation and localization in supercoiled dsDNA. We show that the presence of soft sequences increases the number of plectoneme branches and that the edges of the branches tend to be localized at these sequences. We propose an experimental approach to test our results in vitro, and discuss the possible role played by plectoneme localization in the search process of transcription factors for their targets (promoter regions) on the bacterial genome.
\end{abstract}

The conformational properties of double-stranded (ds) DNA molecules are usually modeled by treating these biopolymers as semi-flexible chains with uniform rigidity that can be represented by a single persistence length (rigidity is proportional to persistence length), $l_{p} \simeq$ $50 \mathrm{~nm}$ in physiological conditions (1, 2). This is justified when one is interested in large-scale properties of dsDNA for which one can replace the sequence-dependent distribution of the elastic rigidity by its average over the chain. When one is interested in small and intermediatescale phenomena one has to consider the full sequencedependence of the rigidity. While some studies suggested that the rigidity of bare dsDNA varies across a limited range $40 \mathrm{~nm}<l_{p}<75 \mathrm{~nm}(3$, 4), experiments on cyclization of short dsDNA fragments $(\approx 100 b p)$ reported much higher cyclization ratios than expected (5, 6). This led to the proposal of DNA kinks - pointlike highly flexible domains (7) - perhaps due to formation of "DNA bubbles" (8, 9). Such sequence-dependent rigidity is a property of bare dsDNA and it has been suggested that the effect can be utilized for the design of promoter sequences in order to control the DNA binding affinity of transcription factors that are sensitive to DNA bendability (10). It may also arise as the consequence of the binding of proteins to specific DNA sequences; indeed, in vivo DNA is partially covered by proteins that affect its flexibility (3, 11,14) and/or its local curvature (15, 16). For example, RecA bacterial proteins polymerize along DNA to give an effective persistence length of hundreds of $\mathrm{nm}$ to the RecA-dsDNA complex (3, 14). Other positivelycharged proteins (e.g., HMGB) and polyamines, increase DNA's flexibility significantly (11, 17, 18). Thus, the variability of DNA rigidity may be even higher in vivo than that of bare DNA in vitro.

In this work we study the interplay between local rigidity and plectoneme localization in supercoiled dsDNA. When circular DNA is subjected to sufficiently large torsional stress, the minimization of the free energy yields strongly-writhed conformations known as plectonemes (see e.g. Fig. 11). We show that the number of plectonemic branches and the locations of the edges (end loops of the branches in Fig. 11) are affected by nonuniform DNA rigidity. This applies not only to circular chains (e.g., bacterial and mitochondrial DNA, and DNA

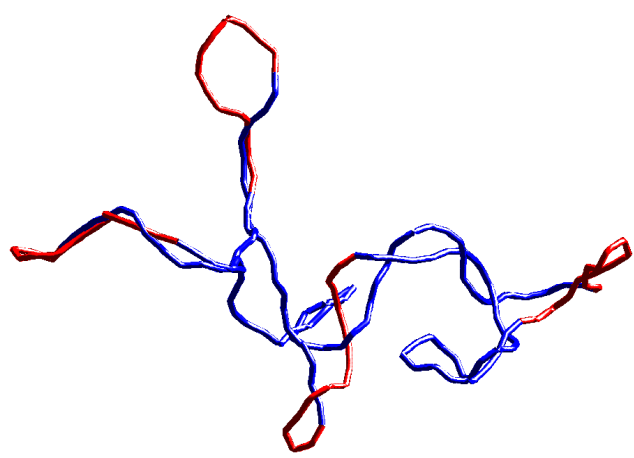

FIG. 1. (color online) Representative conformation of a supercoiled chain of length of $5400 b p$ with a pair of low and high rigidity values $\left(l_{p}^{(l)}, l_{p}^{(h)}\right)=(55 \mathrm{~nm}, 75 \mathrm{~nm})$. Here $l_{p}^{(l)}$ occupies $1 / 3$ of the chain and is divided into 4 equally-spaced domains of identical size, $l_{p}^{(h)}$ occupies the remaining $2 / 3$ of the chain, and the superhelical density is $\sigma=-0.05$. The red segments represent the soft domains, while the blue ones correspond to the stiffer ones. The end loops of the plectonemes tend to be localized at the soft domains.

plasmids), but to topologically-constrained linear dsDNA molecules as well, such as eukaryotic chromosomes that are attached to the nuclear lamina.

We performed Metropolis Monte-Carlo (MC) simulations of topology-conserving worm-like rod (WLR) model that accounts for bending and twist elasticity of dsDNA. A circular dsDNA molecule was modeled as a closed chain of $N$ segments of length $30 \mathrm{bp} \simeq 10 \mathrm{~nm}$ each. The twist persistence length of the chain was taken to be $l_{t w}=$ $74 \mathrm{~nm}$ (corresponding to $\tilde{l}_{t w}=7.4 \mathrm{in}$ units of segment length). The effective diameter of dsDNA was assumed to be $5 \mathrm{~nm}$, taking into account the screened electrostatic repulsion at physiological conditions $(19,20)$. The degree of supercoiling of DNA was characterized by the superhelix density $\sigma$ that is proportional to the amount of torsional stress per unit length of the molecule (20, 21). Bacterial cells have an enzymatic mechanism that keeps the superhelix density of the genome at an almost constant value, typically in the range $-0.03<\sigma<-0.09$ that corresponds to somewhat unwound DNA $(21,22)$ and, 


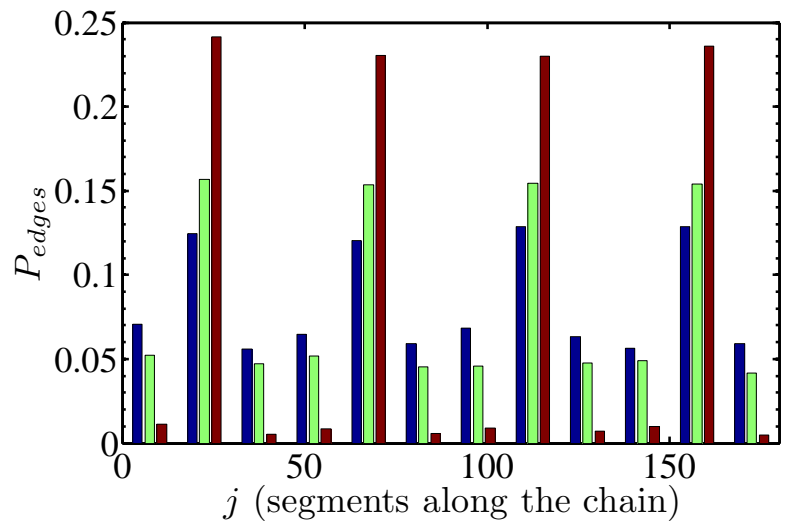

FIG. 2. (color online) Histogram of the locations of the centers of plectoneme edges (end loops), $P_{\text {edges }}$, for a circular chain with $\sigma=-0.06$ and 4 soft domains located around the 23rd, 68th, 113rd and 158th segments. The rigidities correspond to the pairs $\left(l_{p}^{(l)}, l_{p}^{(h)}\right)=(40 \mathrm{~nm}, 55 \mathrm{~nm})$ (blue), $(55 \mathrm{~nm}, 75 \mathrm{~nm})$ (green), and $(50 \mathrm{~nm}, 200 \mathrm{~nm})$ (red).

unless stated otherwise, we assumed that $\sigma=-0.06$. For more details about the energy form used for accepting/rejecting the Metropolis MC steps see Sec. S1 in the SI.

In the simulations we used the pivot (or crankshaft) moves described in detail in ref. (5). The angle of rotation around the pivot was tuned in order to achieve the desired acceptance rate of $50 \%$. To make sure that the simulation was not stuck in a specific plectonemic conformation, we used the linking number inversion method described in ref. (24). We ran our simulations for $5400 \mathrm{bp}$ long chains with segments of two different rigidities distributed along the chain such that most of the chain had a higher rigidity $\left(l_{p}^{(h)}\right)$, and a smaller part of the chain had a lower rigidity $\left(l_{p}^{(l)}\right)$. For each chain conformation taken into account, we determined the number and the locations of plectoneme edges (end loops). The algorithm used for this analysis is described in the SI. The chains in the simulations were unknotted and we made sure that this topology was fixed during the simulation by calculating the Alexander polynomials and Vassiliev invariants after each move, and rejecting topology-changing moves.

We first considered the effect of non-uniform rigidity distribution on plectoneme localization in the case where both the stiff and the soft domains are relatively large (as for example in the RecA-DNA complexes). The soft domain was chosen to occupy $1 / 3$ of the chain and consisted of several domains of identical size, equally-spaced along the contour. In order to model sequence-dependent rigidity of bare dsDNA we took $\left(l_{p}^{(l)}, l_{p}^{(h)}\right)=(40 \mathrm{~nm}, 55 \mathrm{~nm})$ (as in ref. (4)) and $(55 \mathrm{~nm}, 75 \mathrm{~nm})$ (as in ref. (3)). For modeling rigidity induced by protein polymerization along DNA we used $\left(l_{p}^{(l)}, l_{p}^{(h)}\right)=(50 \mathrm{~nm}, 200 \mathrm{~nm})$ (even though polymerization of proteins such as RecA yields rigidities in the range of $600 \mathrm{~nm}-800 \mathrm{~nm}$ (3, 14), the effect of $l_{p}^{(h)} \gg l_{p}^{(l)}$ on the localization of plectoneme edges is already clear for $l_{p}^{(h)}=200 \mathrm{~nm}$ ). In Fig. 2 we plotted a histogram of the locations of the centers of plectoneme edges for a chain with 4 soft domains. Even for the pair with the smallest difference between rigidities $(40 \mathrm{~nm}, 55 \mathrm{~nm})$, there is noticeable localization of the edges to the softer segments of the chain. As the difference between rigidities increases, the localization becomes much more pronounced. For $\left(l_{p}^{(l)}, l_{p}^{(h)}\right)=(50 \mathrm{~nm}, 200 \mathrm{~nm})$, the edges are almost exclusively located in the soft domains. While high superhelix density is a necessary condition for the formation of plectonemes (21), we found that increasing $\sigma$ beyond the plectoneme formation threshold does not have a noticeable effect on the localization of the edges of these branches (see Fig. S5 in the SI).

For chains of uniform rigidity the number of plectonemic branches was shown to increase with $\sigma$ (5). In order to understand how the dependence of the number of chain branches on $\sigma$ is affected by non-uniform rigidity, we compared chains with 4 soft domains with uniform chains of a single persistence length (weighted average of the pair of the corresponding persistence lengths). We observed that for the $(50 \mathrm{~nm}, 200 \mathrm{~nm})$ pair, the presence of soft domains leads to two-fold enhancement compared to homogeneous chains with an average value of $l_{p}=150 \mathrm{~nm}$ (the effect is much weaker both for chains with lower rigidities and those with a smaller difference between higher and lower rigidities- see Fig. S3 in the SI). The origin of this effect is that a chain with $l_{p}=150 \mathrm{~nm}$ has to pay a large bending energy penalty in order to create a tight loop/edge and, as a consequence, the number of edges is small. The presence of soft domains with $l_{p}=50 \mathrm{~nm}$ (even when the stiffness of the rest of the chain is increased), allows the edges to be localized in these domains, thus decreasing the bending energy penalty, and the number of branches increases significantly. The number of plectoneme branches is also affected by the number of soft domains. We calculated the average number of branches as a function of the number of soft domains for $(40 \mathrm{~nm}, 55 \mathrm{~nm}),(55 \mathrm{~nm}, 75 \mathrm{~nm})$ and $(50 \mathrm{~nm}, 200 \mathrm{~nm})$, where the softer part of the chain was divided into $2-6$ domains of equal length, uniformly distributed along the chain (Fig. S4 in the SI). As expected, only a minor effect is observed in the first two cases where the difference between rigidities is small but in the case of large rigidity contrast, the number of edges increases by nearly $50 \%$ as the number of soft domains increases from 2 to 6 .

Next, we proceeded to characterize the effect of DNA kinks (soft local defects) on the localization and the number of plectoneme edges. We simulated $5400 \mathrm{bp}$-long chains with $l_{p}=50 \mathrm{~nm}$, and with $2-6$ kinks at which the persistence length dropped to a value of $4 \mathrm{~nm}$ (about twice the rigidity of ssDNA, (9, 25) ). As can be seen in 


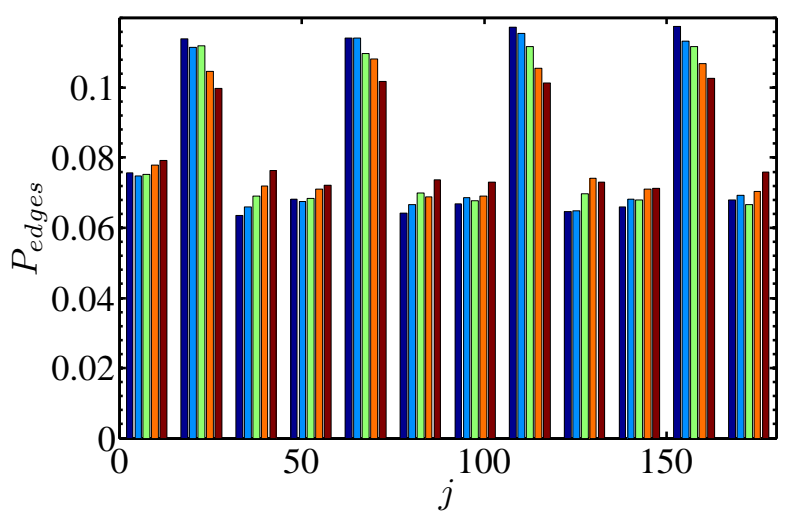

FIG. 3. (color online) Histogram of the locations of plectonemic edges, $P_{\text {edges }}$, for chains with 4 kinks located at the $23 \mathrm{rd}$, 68th, 113rd and 158th dimers, with $\sigma=-0.04$ (dark blue), $\sigma=-0.05$ (light blue), $\sigma=-0.06$ (light green), $\sigma=-0.07$ (orange) and $\sigma=-0.08$ (red).

Fig. 3 for the 4-kink case (and for $2-6$ kinks in Fig. S6 in the SI), plectoneme edges tend to form at the locations of kinks. Another effect observed in Fig. 3 is that in the 4-kink case the fraction of edges that contain kinks decreases monotonicaly with $|\sigma|$, presumably because of the number of branches increases monotonically with $|\sigma|$. In the 6-kink case the decrease in the fraction of kinkcontaining edges is observed only for $|\sigma|=0.07-0.08$ (Fig. S9 in the SI). This concurs with the observation (Fig. S8 in the SI) that while in the 6-kink case the number of edges is only slightly greater than the number of kinks in the chain even for the highest value of $|\sigma|$, in the 4-kinks case the number of edges exceeds the number of kinks already for moderate $|\sigma| \mathrm{s}$.

In Table I and in Figs. S6-S7 in the SI, we show that as the number of kinks, $n_{k i n k}$, increases, the number of branches, $n_{\text {branch }}$, also grows, but this increment is accompanied by an even more rapid increase in the fraction of edges that include a kink, $P_{k i n k}^{(t o t)}$, and in the total number of edges containing a kink, $n_{\cap}$. Even though plectonemes have branches even in the absence of kinks, when supercoiled DNA contains kinks, the edges of the branches are localized preferentially at the kinks; this reduces the bending energy penalty for creating edges and promotes the formation of new branches.

As expected from experience with linear worm-like chains, the presence of a kink affects the local fluctuations of the chain in which it is imbedded. This effect is clearly observed in Fig 4(a) where we compare the distribution of the end-to-end distance $d$ of a short DNA sequence (in a long circular chain) of uniform rigidity, with that of a DNA sequence of the same length and rigidity but containing a kink at its center. Since the length of the sequence is only twice the persistence length of dsDNA,

\begin{tabular}{cccc}
\hline \hline$n_{\text {kink }}$ & $\left\langle n_{\text {branch }}\right\rangle$ & $P_{\text {kink }}^{(\text {tot }}$ & $\left\langle n_{\cap}\right\rangle$ \\
\hline 2 & 5.99 & 0.332 & 1.98 \\
3 & 6.28 & 0.399 & 2.50 \\
4 & 6.41 & 0.441 & 2.63 \\
5 & 6.62 & 0.510 & 3.37 \\
6 & 6.76 & 0.544 & 3.67 \\
\hline
\end{tabular}

TABLE I. The number of branches, $\left\langle n_{\text {branch }}\right\rangle$, the total probability of an edge to contain a kink, $P_{k i n k}^{(t o t)}$, and the total number of edges containing a kink, $\left\langle n_{\cap}\right\rangle=\left\langle n_{\text {branch }}\right\rangle \cdot P_{k i n k}^{(t o t)}$ as a function of the number of kinks in the chain, $n_{k i n k}$, for $5400 b p$ long chains $(\sigma=-0.06)$.

both distributions are asymmetric, with a peak closer to the fully stretched $(100 \mathrm{~nm})$ state. The introduction of the kink shifts the maximum of the distribution from $80 \mathrm{~nm}$ to $70 \mathrm{~nm}$ and results in dramatic enhancement of the ends contact probability $(d \leq 10 \mathrm{~nm})$, an effect that has been invoked to explain the anomalously high cyclization probability of short dsDNA molecules(5) 7). The difference between the distributions becomes much more pronounced in the presence of supercoiling. Since the end loops of plectonemes tend to form around the kinks and the size of the end loop is of order $l_{p}$, we expect the ends of the $100 \mathrm{~nm}$ segment to be preferentially localized in the stem of the plectoneme and the most probable endto-end separation will be below $10 \mathrm{~nm}$. This expectation is fully confirmed in Fig. 4(b) in which we presented the corresponding end-to-end distance distributions of supercoiled DNA for $\sigma=-0.06$ (the histograms for $\sigma$ in the range between 0 and -0.07 are shown in Fig. S10 in the SI). While the distribution for the no-kink case is qualitatively similar to that for $\sigma=0$ (Fig 4(a)), the peak of the distribution of a kink-containing segment is shifted towards contact. In principle, this prediction can be tested by FRET experiments by attaching donor and acceptor molecules to the ends of a short (of order $l_{p}$ ) sequence in a long circular dsDNA molecule, provided that one is able to design a sequence that has a high propensity for forming a kink (26 29). In the absence of supercoiling, the donor and acceptor will be separated by tens of nanometers and there will be no FRET signal. As torsional stress is introduced into the chain, e.g., by raising the concentration of intercalators in the solution (30, 31), plectonemes will form with end loops localized at the kinks and the distance between the donor and acceptor will approach the $6 \mathrm{~nm}$ limit at which energy transfer will take place and a FRET signal will be observed.

This localization effect may also play a significant role in the search process executed by transcription factors (TFs) that attach to specific sequences in promoter regions of DNA. Since bacterial DNA is supercoiled, DNA segments that reside in the stems of the plectonemes 


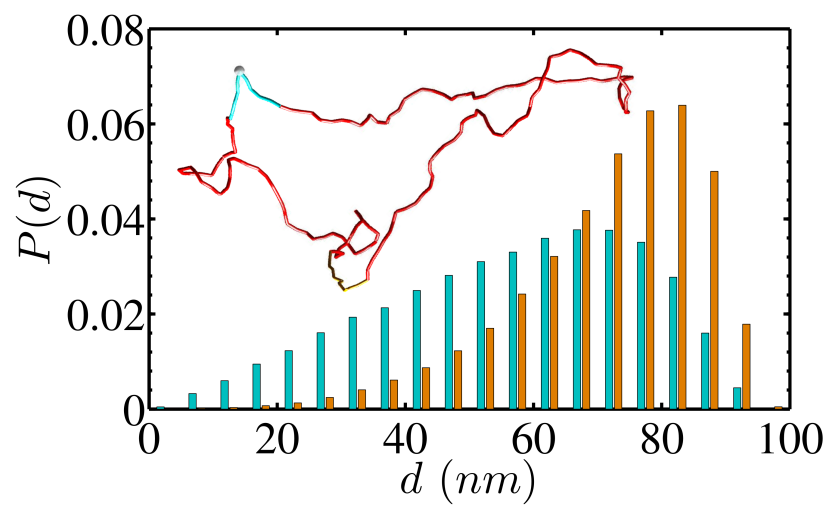

(a)

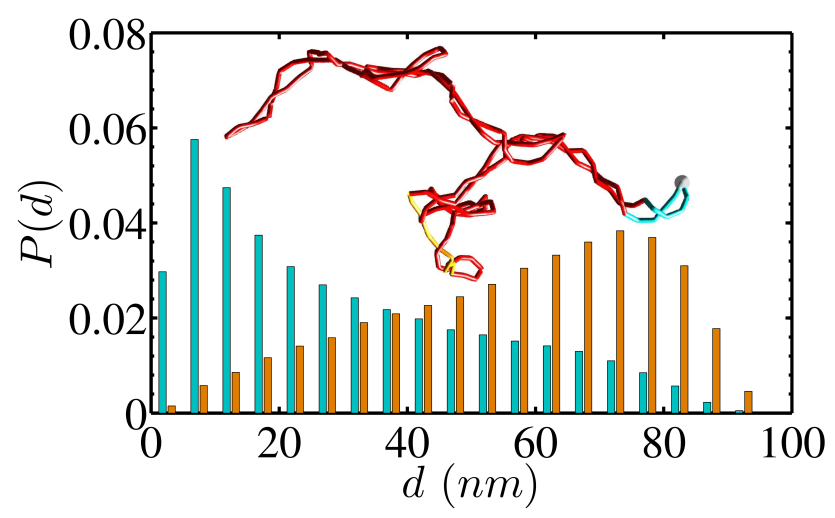

(b)

FIG. 4. (Color online) Histograms of distances of the end points of a $100 \mathrm{~nm}$ long segment in a $5400 \mathrm{bp}$ plasmid containing a kink (light blue) and not containing a kink (orange), for (a) torsionally relaxed $(\sigma=0)$ plasmids and (b) torsionally stressed $(\sigma=-0.06)$ plasmids. Representative DNA conformations are shown in the corresponding figures (red), with a sequence (light blue) containing a kink (sphere). A sequence of the same length but without a kink is shown for comparison (orange.)

are pressed against each other and one expects binding of proteins to these segments to be suppresed. The looped ends of plectonemes are more accessible and, combined with the fact that many bacterial DNA-associated proteins have a higher binding affinity to bent DNA (16, 32, 33), this suggests that TFs may have a higher affinity for end loops of plectonemes. Whether nature utilizes such effects to facilitate the search of TFs for their DNA binding sites depends on whether promoter sites tend to be localized at the edges of plectonemes. Indeed, many bacterial promoters contain TATA-box sequences that are known for their lower rigidity and higher tendency to create DNA bubbles (kinks) (26-29) and, ac- cording to our results, such sequences will tend to nucleate plectonemes and to be positioned at their edges. Finally, we would like to mention that although we are not aware of direct experimental proof of our proposed mechanism of plectoneme localization to low-rigidity DNA sequences, there has been a recent experimental study of the effects of torsional stress on stretched linear dsDNA in which repeated hopping of plectonemes between specific locations along DNA was reported. (34).

\section{ACKNOWLEDGMENTS}

Results obtained in this paper were computed on the biomed virtual organization of the European Grid Infrastructure (http://www.egi.eu). We thank the European Grid Infrastructure and supporting National Grid Initiatives (listed here: http://lsgc.org/en/Biomed:home \#Supporting_National_Grid_Initiatives) for providing the technical support, computing and storage facilities. This work was supported by the I-CORE Program of the Planning and Budgeting committee and the Israel Science Foundation, and by the US-Israel Binational Science Foundation.

(1) P. J. Hagerman, Annual review of biophysics and biophysical chemistry 17, 265 (1988).

(2) W. H. Taylor and P. J. Hagerman, Journal of molecular biology 212, 363 (1990).

(3) J. Leger, J. Robert, L. Bourdieu, D. Chatenay, and J. Marko, Proceedings of the National Academy of Sciences 95, 12295 (1998).

(4) S. Geggier and A. Vologodskii, Proceedings of the National Academy of Sciences 107, 15421 (2010).

(5) T. E. Cloutier and J. Widom, Molecular cell 14, 355 (2004).

(6) T. Cloutier and J. Widom, Proceedings of the National Academy of Sciences of the United States of America 102, 3645 (2005).

(7) P. A. Wiggins, R. Phillips, and P. C. Nelson, Physical Review E 71, 021909 (2005).

(8) J. Yan, R. Kawamura, and J. F. Marko, Physical Review E 71, 061905 (2005).

(9) C. Yuan, E. Rhoades, X. W. Lou, and L. A. Archer, Nucleic acids research 34, 4554 (2006).

(10) M. Levo and E. Segal, Nature Reviews Genetics 15, 453 (2014).

(11) M. C. Williams and L. J. Maher III, Biophysics of DNA-protein interactions (Springer, 2010).

(12) S. M. Rappaport and Y. Rabin, Physical review letters 101, 038101 (2008).

(13) R. Amit, A. B. Oppenheim, and J. Stavans, Biophysical journal 84, 2467 (2003).

(14) E. H. Egelman and A. Stasiak, Journal of molecular biology 191, 677 (1986). 
(15) S. Jones, P. van Heyningen, H. M. Berman, and J. M. Thornton, Journal of molecular biology 287, 877 (1999).

(16) J. Stavans and A. Oppenheim, Physical biology 3, R1 (2006).

(17) A. Podesta, M. Indrieri, D. Brogioli, G. S. Manning, P. Milani, R. Guerra, L. Finzi, and D. Dunlap, Biophysical journal 89, 2558 (2005).

(18) R. S. Hegde, Annual review of biophysics and biomolecular structure 31, 343 (2002).

(19) V. V. Rybenkov, A. V. Vologodskii, and N. R. Cozzarelli, Nucleic acids research 25, 1412 (1997).

(20) A. Vologodskii and V. V. Rybenkov, Physical Chemistry Chemical Physics 11, 10543 (2009).

(21) A. V. Vologodskii and N. R. Cozzarelli, Annual review of biophysics and biomolecular structure 23, 609 (1994).

(22) W. R. Bauer, Annual review of biophysics and bioengineering 7, 287 (1978).

(5) A. V. Vologodskii, S. D. Levene, K. V. Klenin, M. FrankKamenetskii, and N. R. Cozzarelli, Journal of molecular biology 227, 1224 (1992).

(24) S. Medalion and Y. Rabin, The Journal of Chemical Physics 140, 205101 (2014).

(25) M. Murphy, I. Rasnik, W. Cheng, T. M. Lohman, and T. Ha, Biophysical Journal 86, 2530 (2004).
(26) P. T. McNamara, A. Bolshoy, E. N. Trifonov, and R. E. Harrington, Journal of Biomolecular Structure and Dynamics 8, 529 (1990).

(27) J. L. Kim, D. B. Nikolov, and S. K. Burley, Nature 365, 520 (1993).

(28) M. Peyrard, S. Cuesta-Lopez, and D. Angelov, Journal of Physics: Condensed Matter 21, 034103 (2009).

(29) S. Cuesta-Lopez, D. Angelov, and M. Peyrard, EPL (Europhysics Letters) 87, 48009 (2009).

(30) W. Bauer and J. Vinograd, Journal of molecular biology 33, 141 (1968).

(31) T. Steck, G. Pruss, S. Manes, L. Burg, and K. Drlica, Journal of bacteriology 158, 397 (1984).

(32) J. Kim, C. Zwieb, C. Wu, and S. Adhya, Gene 85, 15 (1989).

(33) S. Medalion and Y. Rabin, The Journal of chemical physics 136, 025102 (2012).

(34) M. Van Loenhout, M. de Grunt, and C. Dekker, Science 338, 94 (2012).

(1) F. B. Fuller, Proceedings of the National Academy of Sciences 68, 815 (1971).

(2) J. H. White, American Journal of Mathematics , 693 (1969).

(3) S. Medalion, S. M. Rappaport, and Y. Rabin, The Journal of chemical physics 132, 045101 (2010).

(4) K. Klenin and J. Langowski, Biopolymers 54, 307 (2000).

\section{SUPPORTING INFORMATION}

\section{The Energy Form for the MC Process}

In a discrete worm-like rod (WLR) model that accounts for both the bending diversity and twisting elasticity of the DNA, the energy of a chain (in units of $k_{B} T$ ) with $N$ segments is given by:

$$
\frac{E_{W L R}}{k_{B} T}=\sum_{n=1}^{N}\left[\frac{\tilde{l}_{p, n}}{2}\left(\tilde{\kappa}_{n}\right)^{2}+\frac{\tilde{l}_{t w}}{2}\left(\delta \tilde{\omega}_{t w, n}\right)^{2}\right],
$$

where $\tilde{l}_{p, n}$ is the dimensionless bending persistence length corresponding to the $(n, n+1)$ dimer of segments, $\tilde{l}_{t w}$ is the dimensionless twist persistence length (both measured in units of the segment's length $\Delta s$ ), and $\tilde{\kappa}_{n}=2\left[1-\cos \left(\theta_{n, n+1}\right)\right]$ is the dimensionless curvature between the $n$th and the $(n+1)$ th segments. $\delta \tilde{\omega}_{t w, n}=\tilde{\omega}_{t w, n}-\tilde{\omega}_{t w}^{(0)}$ defines the difference between the $n$th twist angle, $\tilde{\omega}_{t w, n}$, and its spontaneous value, $\tilde{\omega}_{t w}^{(0)}$. The twist angle is the sum of the first and the third rotation angles $\left(\tilde{\omega}_{t w, n}=\varphi+\psi\right)$ of the Euler transformation that rotates the $n$th segment to the $(n+1)$ th segment, while the bending angle, $\theta_{n, n+1}$, is the second Euler rotation angle.

Topologically, supercoiled dsDNA could be described as a closed chain made of two strands infinitesimally closed to each other. This kind of a chain obeys two topological constraints, i.e., the closure of each of its strands. Another description for these topological constraints is given in terms of the closure of the center-line of the chain, and the closure of the cross-sectional plane. These two constraints are fully accounted by the Fuller-White relation (1 13 ):

$$
L k=W r+T w .
$$

where the writhe $W r$ is a measure of the deviation of the center-line from planarity, and the twist $T w$ is proportional to the the sum (in the discrete description) over all the twist angles along the chain. The continuum expressions for $T w$ and $W r$ are given e.g., in (3). In their discrete version they take the forms:

$$
T w=\frac{1}{2 \pi} \sum_{n} \tilde{\omega}_{t w, n}
$$

and:

$$
W r=\frac{1}{4 \pi} \sum_{n, m} W_{n, m}
$$


where $W_{n, m}$ is the discrete pair-of-segments contribution to the $W r$, calculated e.g., in (4) ( $W r$ depends only on the coordinates of the center-line of the chain).

For a circular chain, $L k$ is a topological invariant (an integer) that does not change as long as the topology of the chain is maintained. A supercoiled dsDNA is overwound or unwound depending whether its $L k$ is higher or lower than the preferred value of overall twist, $T w^{(0)}=1 / 2 \pi \sum_{n} \tilde{\omega}_{t w}^{(0)}=N \tilde{\omega}_{t w}^{(0)} / 2 \pi$. When the value of this difference, $\Delta L k \equiv L k-T w^{(0)}$ differs from zero, the chain becomes torsionally stressed and attempts to minimize its total free energy by transforming some of its twist energy into bending energy and, when a critical value of torsional stress is reached, plectonemes appear in the chain.

Since the twist angles of different segments are independent, one may average the twist along the chain by calculating $\left\langle\delta \tilde{\omega}_{t w, n}\right\rangle \simeq 2 \pi T w / N=2 \pi(L k-W r) / N$, where $L k$ is a pre-determined topological constant, and the $W r$ is calculated using the center-line coordinates of the chain. The resulting twist energy, using $\Delta T w \equiv T w-T w^{(0)}=\Delta L k-W r$, is then given by:

$$
E_{T} \simeq \frac{2 \pi^{2} \tilde{l}_{t w}}{N} \Delta T w^{2}=\frac{2 \pi^{2} \tilde{l}_{t w}}{N}(\Delta L k-W r)^{2} .
$$

Thus, the twist energy in our simulations is obtained by using only the coordinates of the center-line of the chain for calculating $W r$, and plugging this value of $W r$ (and the constant $\Delta L k$ ) into Eq. S5 to calculate $E_{T}$.

\section{Methods for Identifying Plectoneme Edge Location}

The number and locations of plectoneme edges (end loops) were determined by two methods. In the first analysis we used the method of calculation of local writhe, $W r^{(l o c)}$ described in ref (5). Although $W r$ of a chain is strictly defined only for closed chains, one may calculate the Gauss double integral along shorter linear segments of length $l$ along the chain to obtain the local writhe associated with the $j$ th monomer, $W r^{(l o c)}(j)$. As shown in Fig. S1, the function $W r^{(l o c)}(j)$ has maxima at end loops (edges) of the plectoneme, and when the value of such a maximum exceeds a threshold, $W r^{(\text {threshold })}$ (the horizontal black dashed line in the figure), this monomer is marked as an edge of the plectoneme (a sphere on the chain in the figure). The height of the peaks of $W r^{(l o c)}$ depends both on the length $l$ of the segments for which we calculate the local writhe and on the superhelix density $\sigma$, since higher $|\sigma| \mathrm{s}$ yield tighter loops resulting in higher local writhe values. Since we want the same threshold value, $W r^{\left({ }^{(t h e s h o l d ~}\right)}$, to distinguish between edges to other segments for different $\sigma \mathrm{s}, l$ must compensate for the $\sigma$-dependence of the heights of the peaks. While in ref (5) the authors flattened the conformations to nearly planar ones before calculating the number of edges, we wanted to analyze the three dimensional structures of the plectonemes, and hence, we used a different function for $l(\sigma)$ and a different value of $W r^{(\text {threshold })}$. In our simulations the choice $l=18 l_{p} /(1+80|\sigma|)$ yielded about the same average heights for the peaks when varying $\sigma$, and $W r^{\text {(threshold })}=0.75$ was found to be an appropriate value to identify the edges from other segments.

For our second analysis we started from the $j$ th segment, and measured the distance $D^{j}(1)$ between the $(j+1)$ th and $(j-1)$ th segments, the distance $D^{j}(2)$ between $(j+2)$ th and $(j-2)$ th segments, and in general, the distance $D^{j}(m)$ between $(j+m)$ th and $(j-m)$ th segments. Plotting $D$ for different $j$ s along the chain for a specific realization one gets curves such as in Fig. S2. If $j$ is a segment somewhere on the stem of the plectoneme, $D$ will increase almost linearly with $m$ as demonstrated, e.g., by the violet curve in Fig. S2, whereas $j$ corresponding to a segment in the center of an end loop will behave differently, i.e., it will slightly increase and then will oscillate with a minimal value very close to the (excluded) diameter of the chain, $d$. Running along specific plectonemic conformation $(j=1,2 \ldots)$, we identified the segments that yield local minimum of the sum of distances $\sum_{m=1}^{m_{\text {cut }}} D^{j}(m)$ (for $m_{\text {cut }}=12$ and accepted only minima under a threshold value of 20 in units of $\Delta s$ ) as the segments in the center of a loop. An advantage of this analysis is that by examining the shape of $D^{j}(m)$ for the center points of an edge, one may determine the lengths of the branches as well, since when the two chain parts depart from each other (for some $m$ ), there is an abrupt change in the slope of $D^{j}(m)$. However, for our purposes the two methods of analysis agreed in more than $95 \%$ of the cases.

(1) F. B. Fuller, Proceedings of the National Academy of Sciences 68, 815 (1971).

(2) J. H. White, American Journal of Mathematics , 693 (1969).

(3) S. Medalion, S. M. Rappaport, and Y. Rabin, The Journal of chemical physics 132, 045101 (2010).

(4) K. Klenin and J. Langowski, Biopolymers 54, 307 (2000). 


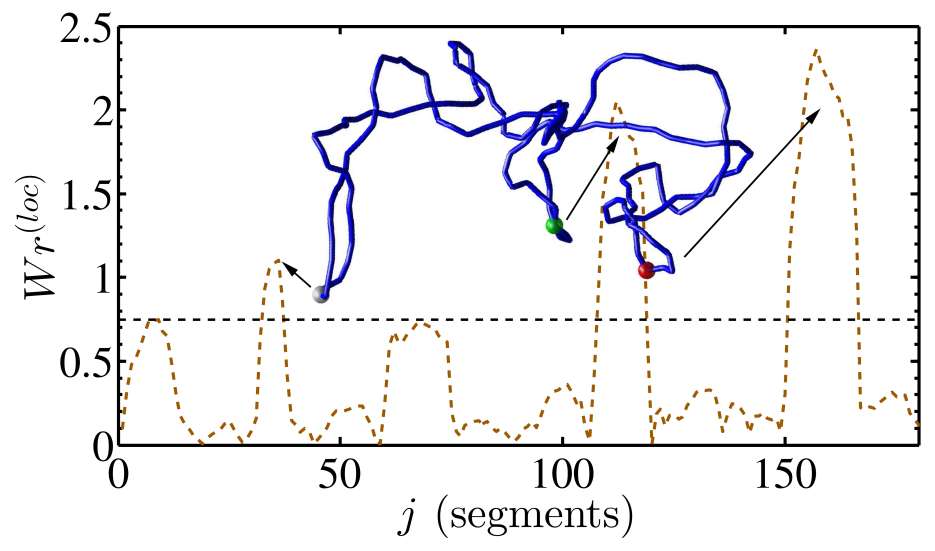

FIG. S1. (Color online) $W r^{(l o c)}$ along the chain, for the specific realization plotted in the figure. The spheres at the edges correspond to the locations of the peaks of $W r^{(l o c)}$.

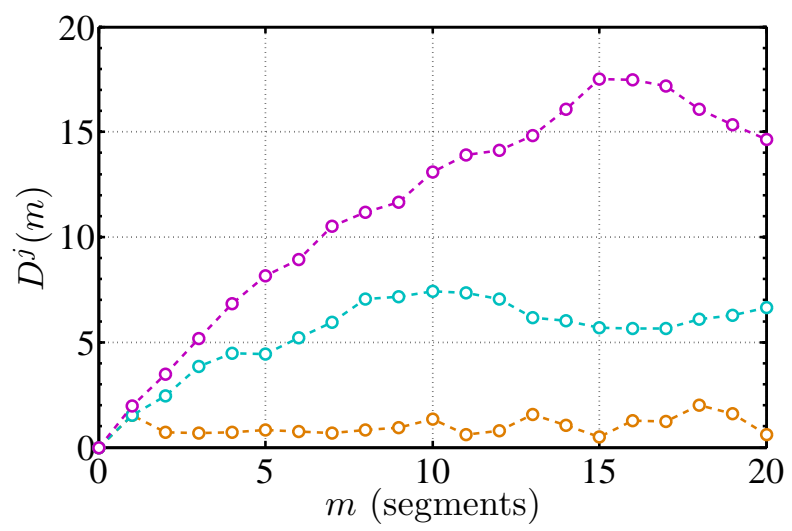

FIG. S2. (Color online) Typical realization of $D^{j}(m)$ starting from different $j$ s along the chain: center of an edge (orange), middle of a branch body (violet), and a point somewhere in between (light blue).

(5) A. V. Vologodskii, S. D. Levene, K. V. Klenin, M. Frank-Kamenetskii, and N. R. Cozzarelli, Journal of molecular biology 227, 1224 (1992). 


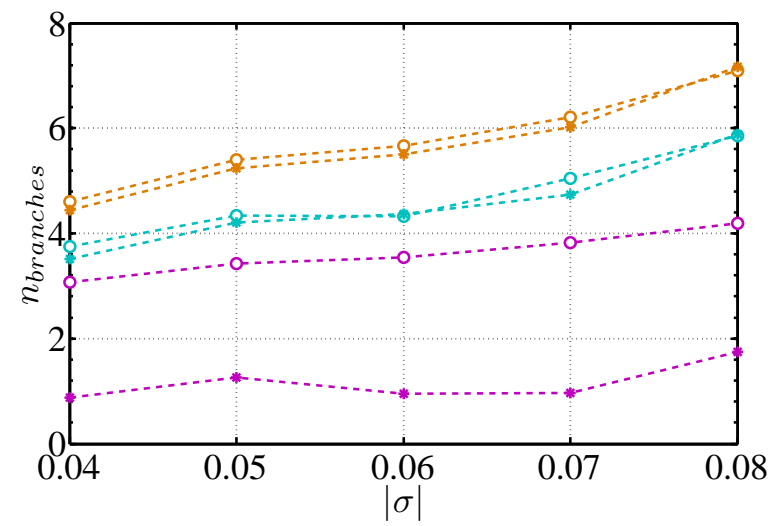

FIG. S3. (color online) Average number of branches of the plectonemes as a function of the absolute value of $\sigma$, for chains with high/low rigidities compared to chains with a single rigidity given by the weighted average of the matching pair of persistence lengths: $\left\langle l_{p}\right\rangle=\frac{1}{3} l_{p}^{(l)}+\frac{2}{3} l_{p}^{(h)}$. The pair rigidities and the corresponding weighted averges are: $\left(l_{p}^{(l)}, l_{p}^{(h)}\right)=(40 \mathrm{~nm}, 55 \mathrm{~nm})($ orange dashed line with circles) and 50 $\mathrm{nm}$ (orange solid line with asterisks); (55nm, 75 $\mathrm{nm}$ ) (blue dashed line with circles) and 68.3nm (blue solid line with asterisks); (50nm, 200 nm) (violet dashed line with circles) and 150nm (violet solid line with asterisks).

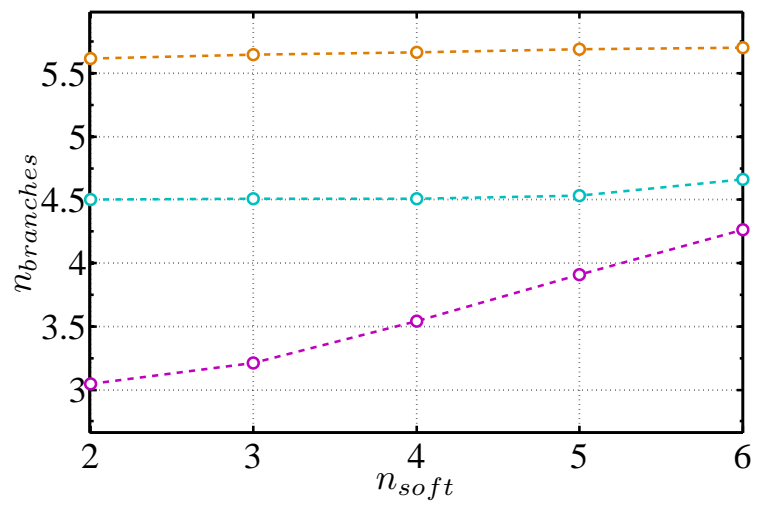

FIG. S4. (color online) Average number of plectoneme branches as a function of the number of soft domains for chains with persistence-length pairs: $\left(l_{p}^{(l)}, l_{p}^{(h)}\right)=(40 \mathrm{~nm}, 55 \mathrm{~nm})$ in orange, $(55 \mathrm{~nm}, 75 \mathrm{~nm})$ in blue, and $(50 \mathrm{~nm}, 200 \mathrm{~nm})$ in violet. 


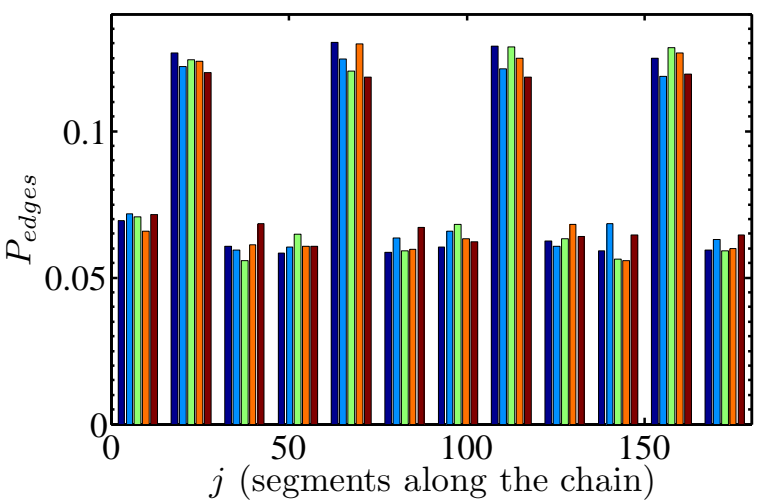

(a)

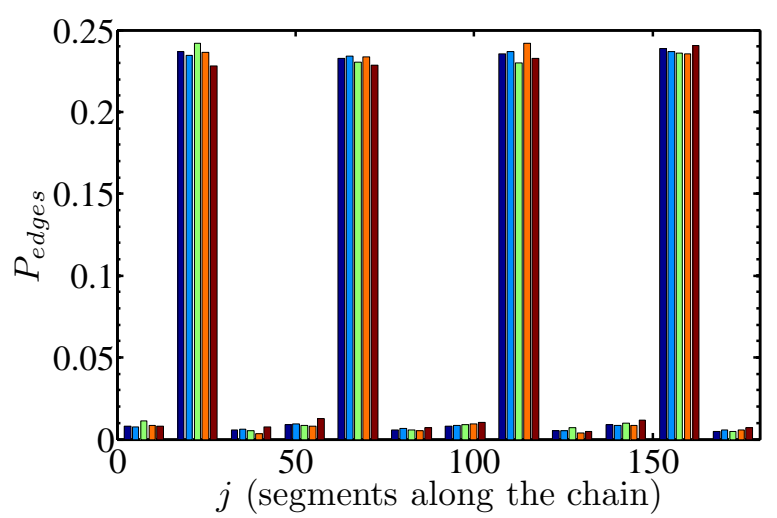

(b)

FIG. S5. (Color online) Histograms of the locations of the centers of the plectonemic edges (loops), $P_{\text {edges }}$, for a circular chain with 4 soft regions located around the 23rd, 68th, 113rd and 158th segments for: $\sigma=-0.04$ (dark blue), $\sigma=-0.05$ (light blue), $\sigma=-0.06$ (light green), $\sigma=-0.07$ (orange) and $\sigma=-0.08$ (red). (a) $\left(l_{p}^{(l)}, l_{p}^{(h)}\right)=(40 \mathrm{~nm}, 55 \mathrm{~nm}),(\mathrm{b})\left(l_{p}^{(l)}, l_{p}^{(h)}\right)=$ $(50 \mathrm{~nm}, 200 \mathrm{~nm})$. Note that the probability of localization of the edges to the kinks doesn't change significantly with $\sigma$. 


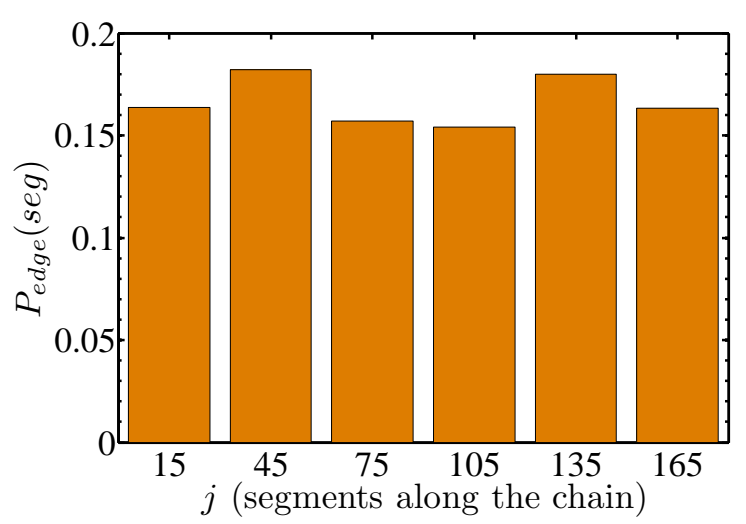

(a)

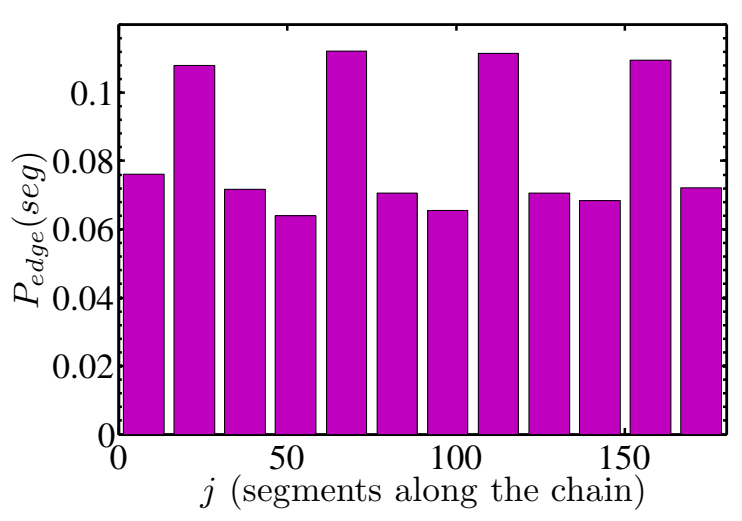

(c)

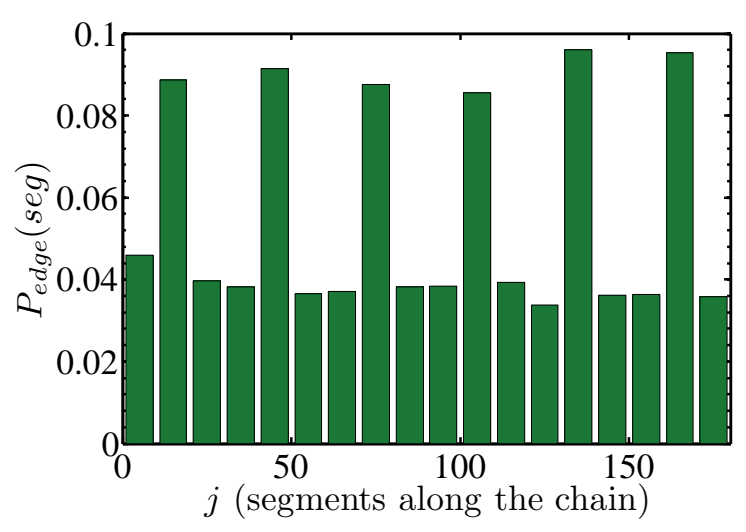

(e)

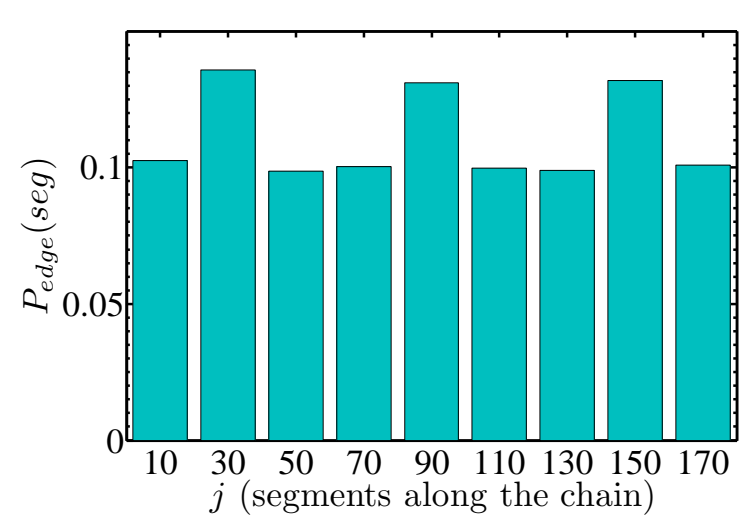

(b)

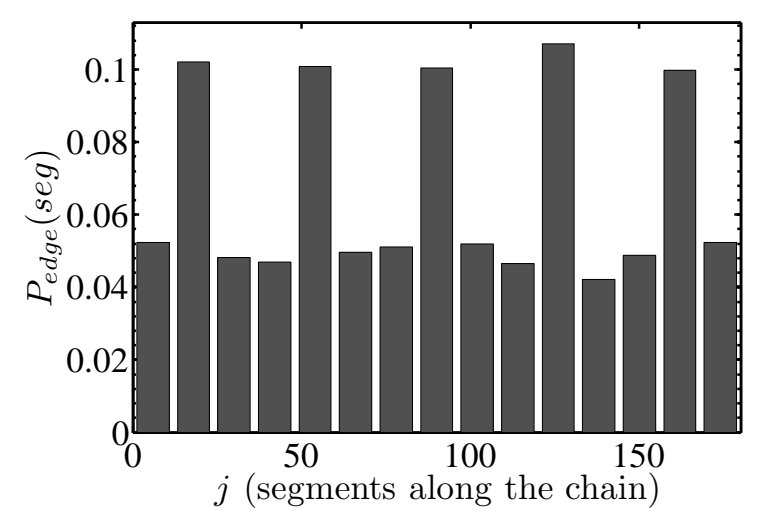

(d)

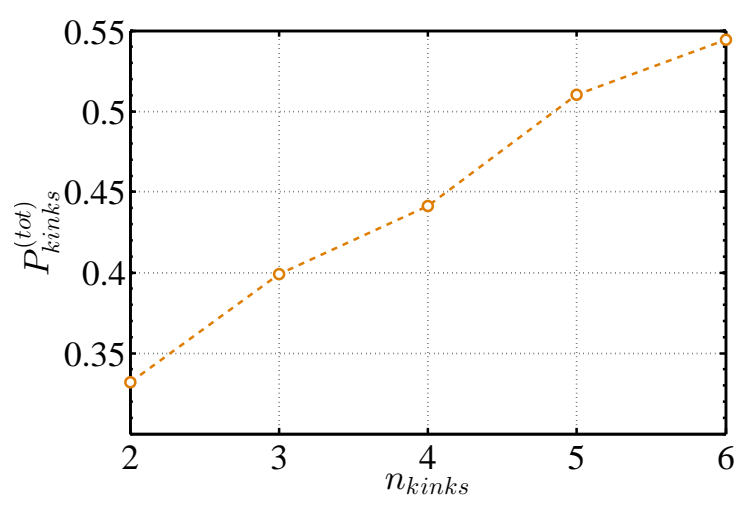

(f)

FIG. S6. (Color online) Histograms of the locations of the centers of the plectoneme edges, $P_{\text {edges }}$, for a circular chain of $L \simeq 5400 \mathrm{bp} \simeq 180 \mathrm{seg}$ with point-like kinks with $l_{p}=4 \mathrm{~nm}$ where the rest of the chain has $l_{p}=50 \mathrm{~nm}$. (a) two kinks located at $j=45$ and $j=135$ (b) three kinks located at $j=31, j=91$ and $j=151$ (c) four kinks located at $j=23, j=68, j=113$ and $j=158$ (d) five kinks located at $j=19, j=55, j=91, j=127$ and $j=163$ (e) six kinks located at $j=16, j=46, j=76$, $j=106, j=136$ and $j=166$ (f) the overall probability for an edge to contain a kink as a function of the number of kinks. The existence of higher number of kinks effectively "sucks" the probability from the other parts of the chain, and as can be seen in (a)-(e) the difference between the probabilities of kink-containing and kink-free edges increases. 


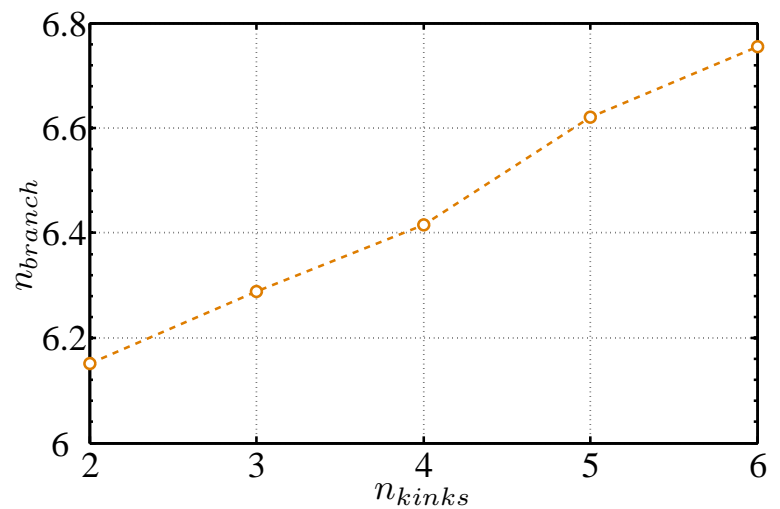

FIG. S7. (color online) The mean number of plectoneme branches as a function of the number of kinks for $5400 b p$-long chains with $l_{p}=50 \mathrm{~nm}$ for the chain and $l_{p}=4 \mathrm{~nm}$ for the kinks.

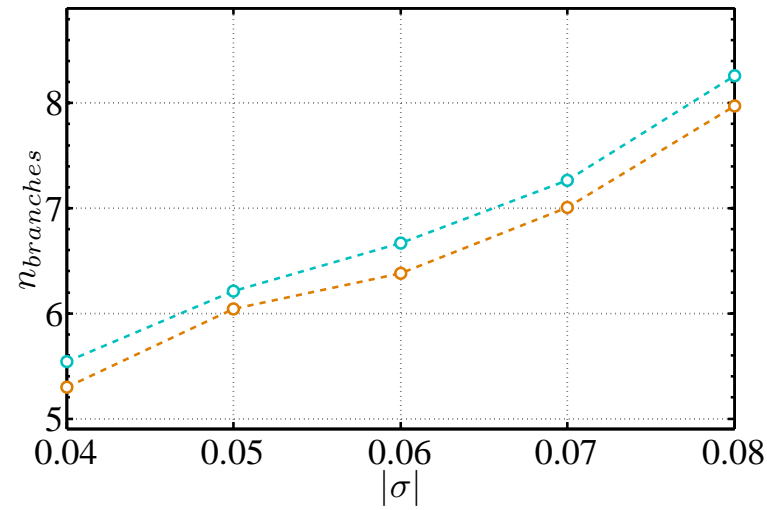

FIG. S8. (color online) The number of branches of the chain, $n_{\text {branch }}$, as a function of $|\sigma|$ for 4-kinks (orange) and 6-kinks (light blue). While for 6-kinks the number of branches is comparable with the number of kinks, for 4-kinks the number of branches exceeds the number of kinks.

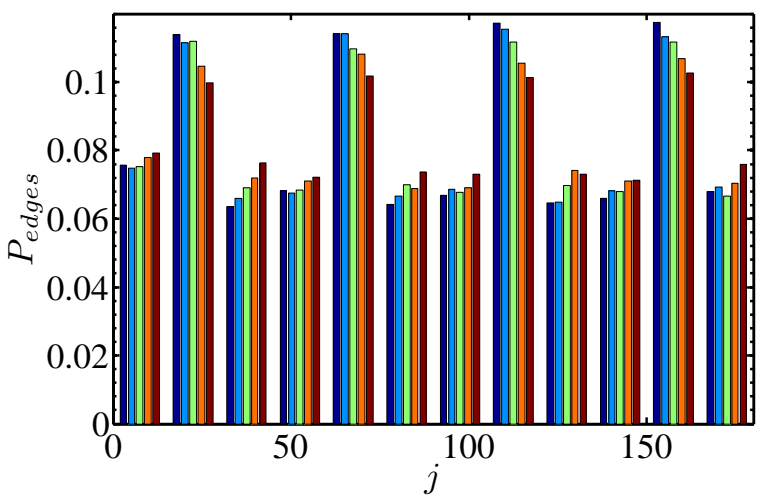

(a)

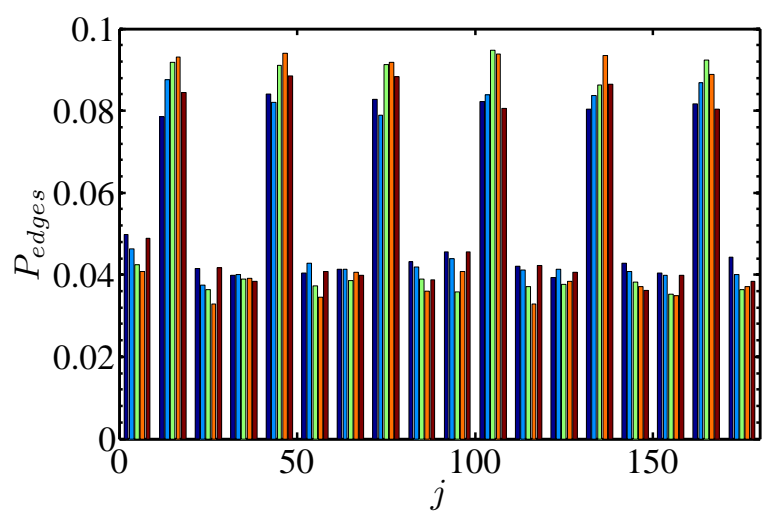

(b)

FIG. S9. (Color online) Histograms of the locations of the plectoneme edges, $P_{\text {edges }}$, for $\sigma=-0.04$ (dark blue), $\sigma=-0.05$ (light blue), $\sigma=-0.06$ (light green), $\sigma=-0.07$ (orange) and $\sigma=-0.08$ (red). (a) 4 kinks located at the 22nd, $62 \mathrm{nd}, 112 \mathrm{nd}$ and 152nd dimers and (b) 6 kinks located at $j=16, j=46, j=76, j=106, j=136$ and $j=166$. 


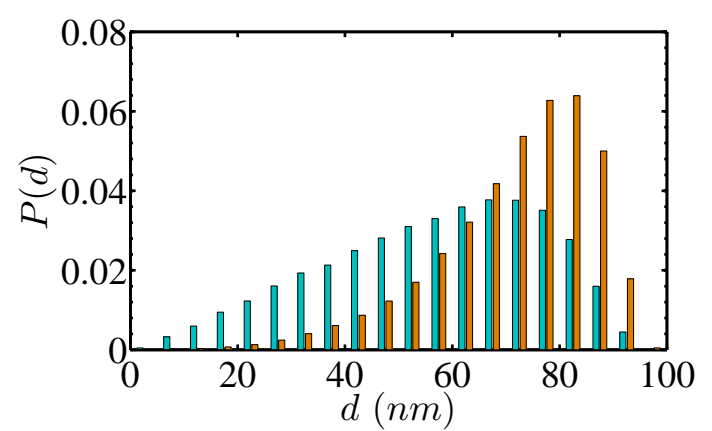

(a)

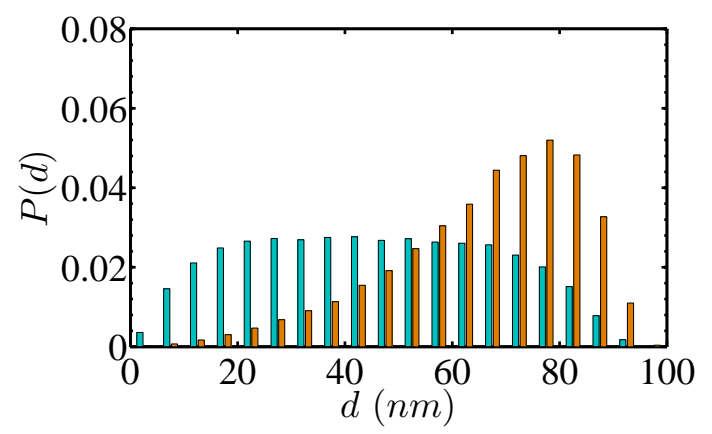

(c)

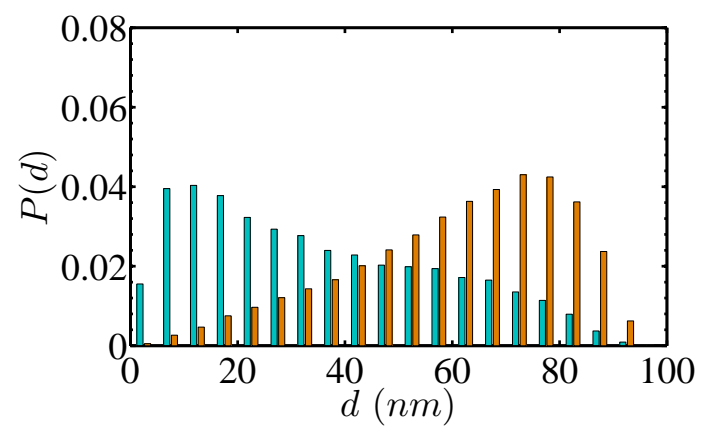

(e)

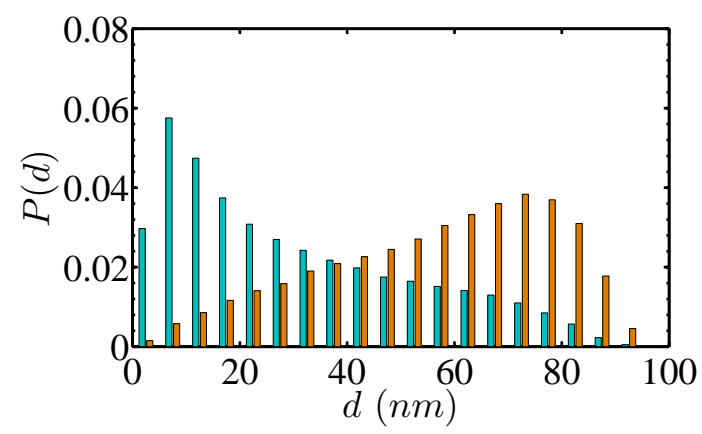

(g)

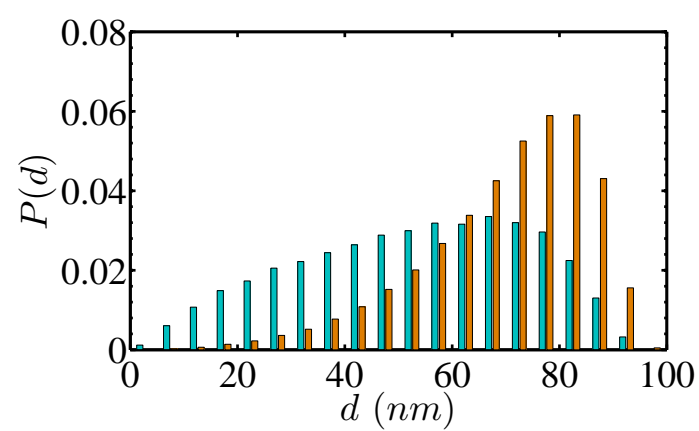

(b)

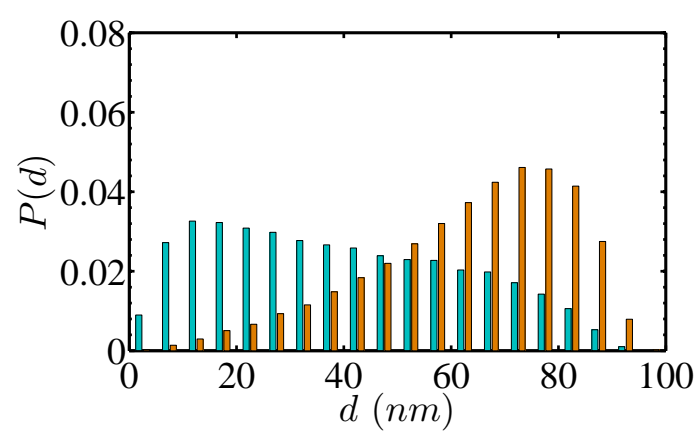

(d)

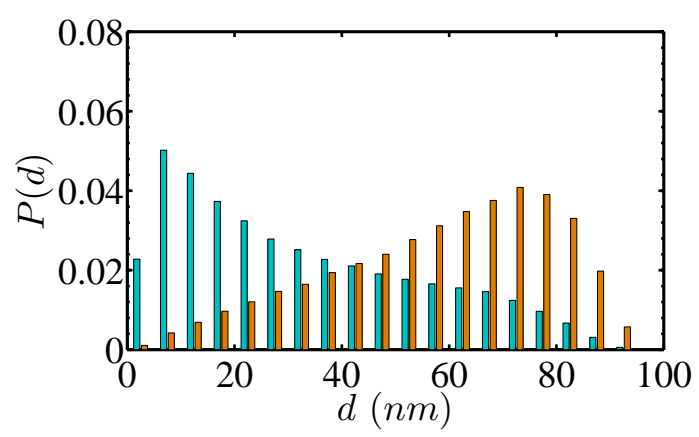

(f)

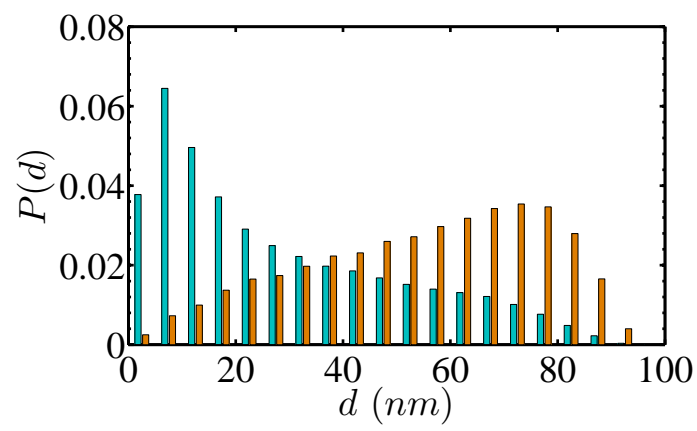

(h)

FIG. S10. (Color online) Histograms of distances of the end points of a 100 $\mathrm{nm}$ long segment in a $5400 \mathrm{bp}$ plasmid containing a kink (light blue) and not containing a kink (orange), for (a) $\sigma=0$ (b) $\sigma=-0.01$ (c) $\sigma=-0.02$ (d) $\sigma=-0.03$ (e) $\sigma=-0.04$ (f) $\sigma=-0.05(\mathrm{~g}) \sigma=-0.06(\mathrm{~h}) \sigma=-0.07$. The shift of the maximal probability to short distances, and the significant enhancement of probability for the shortest distances are clearly observed for segments containing a kink. 